\title{
Excimer laser ophthalmic surgery: evaluation of a new technology
}

\author{
David A Infeld, John G O’Shea
}

\begin{abstract}
Summary
The aim of this article is to provide information and an overview of the potential risks and benefits of excimer laser surgery, a new and promising technique in ophthalmic surgery. Although this review concentrates on the use of the laser for refractive purposes, novel therapeutic techniques are also discussed. It is hoped that this will enable general practitioners, optometrists and physicians to provide appropriate advice and counselling for patients.
\end{abstract}

Keywords: excimer laser; ophthalmic surgery

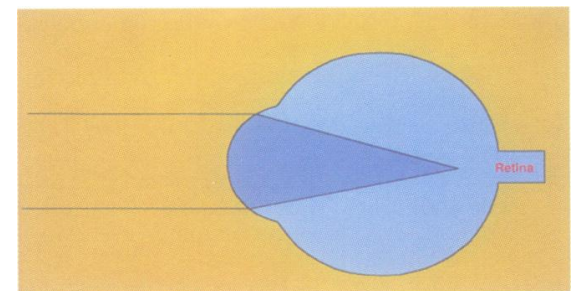

Figure 1 In myopia, the principal focus of the eye is anterior to the retina. The cornea is the principal refractive surface of the eye and its shape is altered by the excimer laser. The eye has a functional dioptric power too high for its length because the eye ball is long; alternately, the lens or cornea act as a more powerful converging lenses than is physiologically appropriate
Birmingham and Midland Eye Centre, Dudley Road, Birmingham B18 7QU,

UK

D A Infeld

J G O'Shea

Correspondence to Mr J G O'Shea

Accepted 10 March 1998
The aim of refractive surgery is to eliminate the need for spectacles or contact lenses or to reduce the strength of the prescription. ${ }^{1}$ The most common cause of visual impairment is refractive error rather than cataracts or age-related macular degeneration. (The World Health Organisation (WHO) definition of blindness is vision less than $3 / 60$ in the better eye with the best available correction; visual disability or impairment is defined as acuity $6 / 18$ to $3 / 60$ with or without spectacle correction.)

Myopia and regular myopic astigmatism are now the commonest indications for excimer laser procedures; laser treatment of hypermetropia is still at best problematic. Modalities used to treat myopia include photorefractive keratectomy (PRK) and laser in situ keratomileusis. ${ }^{2}$ Phototherapeutic keratectomy (PTK) utilises the excimer laser for therapeutic, not refractive purposes. For example, the treatment of recurrent erosion syndrome or of an anterior corneal dystrophy.

\section{Ametropia (refractive error) and common refractive problems}

Emmetropia refers to the normal refractive state. Here, incoming light rays are focused on the retina. The cornea is the principal refractive component of the eye, ${ }^{3}$ providing two-thirds of the eye's refractive power, with the crystalline lens providing the remaining third. The lens can increase its focusing power by changing its shape, enabling near objects to be seen clearly; this is called accommodation. Ametropia, or refractive errors, can be due to hypermetropia, myopia, or astigmatism (figures $1-4) .{ }^{14}$

\section{HYPERMETROPIA}

In hypermetropia (long sightedness), parallel light rays from a distant object are not focused on the retina unless there is some degree of accommodation by the crystalline lens. Hypermetropia is often due to the eye being relatively small. ${ }^{14}$ Hypermetropes often notice tiredness or aching with prolonged reading or other close work due to excessive accommodation. Hypermetropia is neutralised with a convex spectacle lens or contact lens. ${ }^{14}$ Treatment of hypermetropia with the excimer laser is not recommended.

\section{PRESBYOPIA}

Presbyopia, although not a refractive error, refers to the difficulties experienced with prolonged near vision associated with ageing. ${ }^{14}$ It is an almost universal phenomenon, typically noticed at an age of 40 to 45 years and is due to loss of flexibility and power of the crystalline lens. Presbyopic symptoms can, however, develop at an earlier age particularly after excimer laser treatment in a myopic patient. A convex reading lens which increases the dioptric power of the eye treats presbyopia. ${ }^{14}$

\section{MYOPIA}

In myopia (short sightedness; box 1) distance vision is blurred because the light rays are focused anterior to the retina. ${ }^{14}$ Myopia affects about one quarter of the population; it is often due to the eye being relatively large. Myopia usually begins in the first or second decade and stabilises by 20 years of age. Myopia can be neutralised with a concave spectacle lens or contact lens. ${ }^{14}$

Myopic refractive prescriptions are measured in dioptres, the reciprocal of the far point of the patient's vision in metres. For example, a 4-dioptre myope has a far point of $25 \mathrm{~cm}$. Beyond this distance light rays will not be focused onto the retina. The patient will not be able to drive without spectacles. 'High myopia' is myopia above 6 dioptres. It is often associated with structural abnormalities of the eye, for example, macular degeneration and retinal detachment. The excimer laser does nothing to correct these structural abnormalities. 


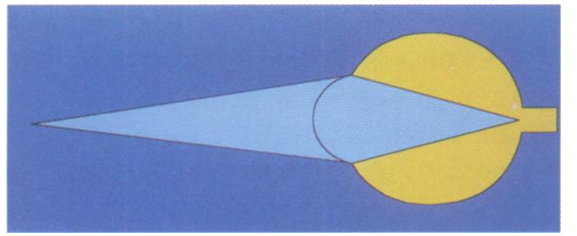

Figure 2 The myopic far point is a the point beyond which light rays cannot be focused on to the retina. Near-parallel rays of light coming from a more distant source cannot be focused on to the retina and hence distance vision is blurred

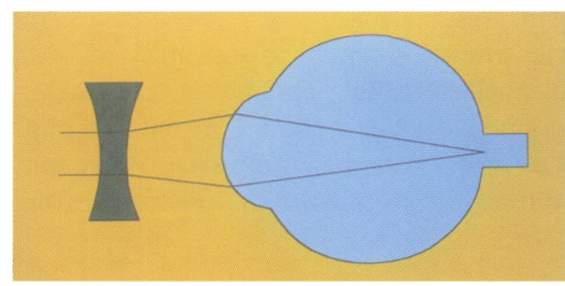

Figure 3 Myopia is corrected by a concave lens. This renders parallel rays of light divergent, effectively decreasing the dioptric power of the myopic eye. Optic prescriptions describe a myopic prescription in terms of dioptres, the reciprocal of the focal length of the lens needed to correct myopia in dioptres, hence a -2.0 dioptre myope requires a diverging, concave lens with a focal length of $50 \mathrm{~cm}$. The excimer laser effectively cuts a concave correction in the cornea, decreasing the dioptric power of the eye

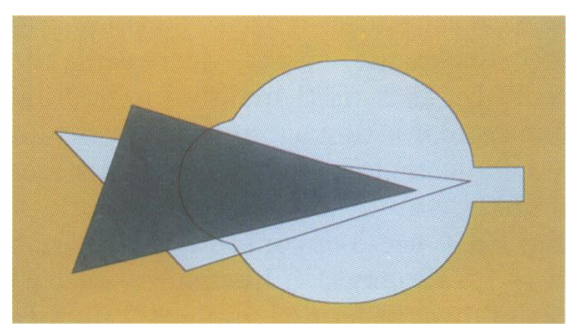

Figure 4 This diagram shows simple, regular myopic astigmatism. In the shaded, vertical meridian the focus of the eye is anterior to the retina whilst in the horizontal, non-shaded meridian the eye focuses on the retina. Regular astigmatism is corrected by cylindrical lenses or by toroidal (toric) lenses which are lenses that incorporate both a cylindrical and spherical component. Modern excimer lasers can cut a complex, toroidal correction into the cornea
ASTIGMATISM

Astigmatism is usually due to a lack of corneal sphericity; circular objects may appear oval. Regular astigmatism is the commonest form. Current refractive surgery aims to treat regular astigmatism. Astigmatism is neutralised with cylindrical or toroidal lenses (figure 4). Current refractive surgery techniques also aim to treat regular myopic astigmatism. ${ }^{14}$

\section{Refractive surgery}

Refractive surgery refers to surgical procedures that alter the focusing power of the eye, usually by changing the shape of the cornea. The main techniques are PRK using an excimer laser, radial keratotomy, and lamellar surgery (such as an intracorneal lens or ring). Intra-ocular surgery (such as corneal graft, clear lens extraction and phakic lens implantation) is usually reserved for more extreme types of refractive error.

INDICATIONS FOR REFRACTIVE SURGERY

Patients often seek refractive surgery for occupational or cosmetic reasons. Some patients may have spectacle or contact lens intolerance. For example, high correction spectacles are heavy and produce peripheral image distortion. ${ }^{14}$ Occasionally patients require refractive surgery to correct a refractive error secondary to previous eye surgery (such as cataract surgery). ${ }^{1-4}$

\section{ASSESSMENT AND COUNSELLING}

It is important to determine the patient's reason for seeking treatment. Patients typically consider undergoing PRK because of intolerance of glasses or contact lenses, for occupational reasons, due to recreational or sporting activities, or for cosmetic reasons. ${ }^{12}$

As with all surgical procedures, informed consent is required. Adequate information should be provided regarding possible complications and other treatment options. Patients require the opportunity to ask questions. The patient needs to be aware that visual acuity will not be improved. A middle aged myope needs to be aware that he or she may require a presbyopic correction following treatment. ${ }^{1}$ Patients should obtain a letter of referral from their general practitioner. The patient's refractive error needs to have stabilised; usually this occurs by 21 years of age.

Counselling should be provided by a refractive surgeon who is experienced with PRK. Educational videos and information leaflets are also very useful. Some patients may be unsuitable because they cannot give a legally valid consent to operation. For example, some patients are unable to understand the concept of probability and are therefore unable to give informed consent. It is inadvisable to ask the patient to sign the consent form during the first consultation, and patients should not be treated immediately after the first consultation.

The issue of presbyopia should be outlined to the patient when appropriate. For example, a 50-year-old myope may require reading glasses following successful PRK. The optical disadvantage created for such a patient by PRK can be simulated with trial frame lenses. ${ }^{13}$

After 60 years of age significant cataract often develops, in which case cataract surgery is more appropriate than corneal refractive surgery.

It is also strongly recommended that other eye should not undergo treatment within a period of 6 months. This time interval, with associated loss of refined stereo vision could, in some patients, lead to occupational difficulties. ${ }^{156}$ The patient should be informed that occupational difficulties also result from the requirement to cease soft contact lens wear for 2 weeks pre-operatively and hard contact lens wear for 6 weeks pre-operatively.

\section{The excimer laser}

The excimer laser is an argon/fluoride laser which emits coherent ultraviolet C light at $193 \mathrm{~nm}$. It is capable of breaking intermolecular valence bond of proteins and other macromolecules without causing thermal damage to adjacent tissue. The selected wavelength also avoids mutagenesis. The laser alters the radius of curvature of the cornea very precisely. The surface created by the laser is both remarkably smooth and geometrically precise. ${ }^{156}$ Healing of the cornea is, however, less predictable and cannot as yet be mathematically modelled (box 2). ${ }^{125-8}$

\section{MECHANISM OF ACTION}

The cornea is usually much steeper in the centre than in the periphery. The central cornea has less refractive power than the peripheral cornea following excimer laser treatment for myopia due to sculpting by the ins.. The cornea absorbs the 


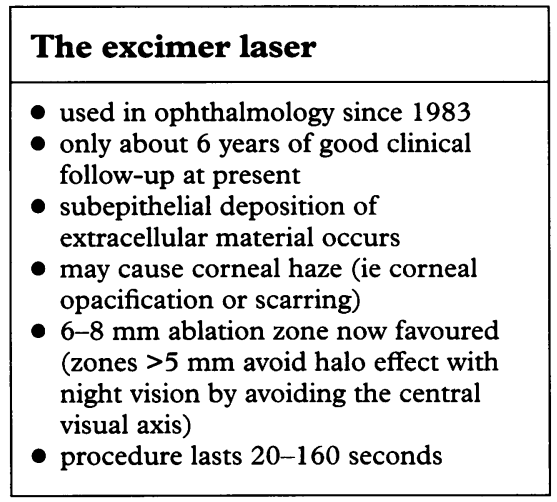

Box 2

\section{Pre-operative preparation for excimer laser}

- visual acuity + refraction

- computerised corneal topography detects subclinical keratoconus and quantifies astigmatism or other refractive error (now regarded as mandatory)

- soft contact lenses are removed 2 weeks prior to procedure and hard contact lenses 6 weeks

Box 3

\section{Postoperative regimen}

- cycloplegics

- analgesics

- lubricants

- antibiotics until epithelium has healed

Box 4

\section{Theoretical and practical problems with treatment of high myopia (>6 dioptre) by PRK}

- over- or under-correction, regression of treatment

- persistent corneal opacity

- irregular astigmatism

- decreased contrast sensitivity

- decrease of 1-3 lines of best corrected acuity on Snellen chart coherent ultraviolet light and is sculpted in an extremely precise fashion, namely, by amounts of $0.5 \mu \mathrm{m}$ (the central corneal thickness is about $500 \mu \mathrm{m}$ ). ${ }^{156-8}$

The long-term clinical significance of corneal shock waves from the excimer laser is unknown. There does not appear to be a significant decrease in the corneal endothelial cell count, critical to the health of the cornea. ${ }^{18}$ The required depth of corneal sculpting is proportional to the degree of myopia to be and/or astigmatism treated. Deep ablation may lead to structural weakness of the cornea. ${ }^{15-9}$

\section{THE LASER PROCEDURE}

The laser procedure is undertaken on an outpatient basis. Important steps in the procedure are as follows:

- the surgeon checks that the patient is suitable for treatment and that the refractive error has been properly determined

- patients require a detailed refractive assessment. Corneal topography prior to the procedure is now regarded as mandatory. It provides a computergenerated map of the surface of the cornea. It can detect corneal warpage from contact lens wear as well as early keratoconus, in which case PRK may not be inadvisable. The surgeon then enters the details of the desired refractive correction into a computer

- the corneal epithelium is removed ${ }^{18}$

- the centration of the laser treatment is checked. The patient needs to maintain fixation. The eye not being treated is covered. The laser treatment itself is completed in less than one minute. ${ }^{8}$

The laser treatment is undertaken using topical anaesthesia. The patient usually experiences some pain for two or three days. ${ }^{10}$ Postoperatively, topical anti-inflammatory agents, antibiotics and oral analgesia are usually prescribed (box 4). An eye patch is worn for one day. ${ }^{810}$ The use of postoperative topical steroids is controversial. ${ }^{11}$ Re-treatment, if necessary, should be delayed for 6-12 months. $^{8}{ }^{9}$

\section{Excimer laser refractive procedures}

PHOTOREFRACTIVE KERATECTOMY (PRK)

In PRK, an excimer laser makes accurate cuts in the cornea, decreasing the refractive power of the anterior corneal surface. It is used mainly for treating myopia and can also be used to treat myopic regular astigmatism. It is important to note that the excimer laser has been used clinically for only a few years; therefore the possible long-term effects remain unknown. ${ }^{18911}$

Currently recommended indications for PRK are a myopia of less than 6 dioptres with little associated astigmatism ( $<1.5$ dioptres). The results of treatment are less predictable for higher degrees of myopia. For example, a 7 dioptre myope is far less likely to be rendered emmetropic than a 2 dioptre myope. However, the 7 dioptre myope may still be delighted with a postoperative refractive error of 2 dioptres. Results may be also operator-dependent with experienced surgeons producing better results than less experienced surgeons (box 5). ${ }^{18-11}$

\section{Topical corticosteroids and PRK}

The long-term effects of topical corticosteroid use on the final refractive results are undocumented, while the effects on corneal haze are also controversial. ${ }^{11}{ }^{12}$

\section{Complications and contraindications of $P R K$}

Problems with PRK include an unpredictable postoperative refractive result, and loss of the desired refractive outcome (regression). ${ }^{9}$ Patients tend to become under-corrected with time, more so with higher degrees of correction. ${ }^{18911}$ Over-correction, ie, hypermetropia, can also occur, particularly with certain excimer laser machines. The period of time required for visual rehabilitation depends on the amount of ametropia corrected and the type of laser machine used (box 6).

Complications of PRK include pain, corneal opacification, blurred vision, glare, impaired night vision, night haloes and astigmatism. ${ }^{1813-16}$ Night haloes, concentric blur circles caused by the edge of the excimer ablation zone, may be so troubling as to affect night driving. This is probably the commonest complication of well planned surgery. In one study, $78 \%$ of patients noticed a halo around lights at night. ${ }^{14}$ This symptom is less likely to occur with treatment zones larger than $4 \mathrm{~mm}$ in diameter. Impaired night vision and glare are still common problems, occurring in nearly $60 \%$ of patients. ${ }^{13} \mathrm{With}$ higher degrees of myopia there is a greater risk of corneal scarring, blurred vision, ametropia and regression. 


\begin{tabular}{|l|}
\hline Contraindications to excimer \\
refractive surgery \\
\hline Absolute contraindications \\
- monocularity \\
- irregular astigmatism \\
past or present corneal Herpes simplex \\
virus infection \\
- concoma \\
- age younger than 21 years \\
- ectatic corneal dystrophies (ie, \\
$\quad$ keratoconus) \\
Relative contraindications \\
- pregnancy \\
- connective tissue disease \\
\hline
\end{tabular}

Box 6
Corneal haze (opacification) often occurs following PRK; it is usually asymptomatic but in severe cases it causes blurred vision. 'Haze' is a euphemism that is used when referring to this opacification. The haze increases to a maximum at about 6 months postoperatively and tends to decrease to some extent after 2 or more years. ${ }^{1817}$

Astigmatism and a prismatic effect can result from decentration of the laser treatment, this is often secondary to poor patient fixation. If the astigmatism is irregular then it cannot be optically neutralised, in which case the vision will be permanently blurred.

One adverse effect of treatment is an artefactual decrease in intra-ocular pressure due to the laser-induced corneal thinning. The diagnosis of glaucoma, a condition more common in myopes, may be unnecessarily delayed. ${ }^{8}$

\section{Subjective results of surgery}

A study conducted by Hamberg-Nystrom and co-workers to evaluate the subjective results of patients who underwent PRK for myopia with excimer laser is typical of results for the procedure quoted in the current literature. ${ }^{18} \mathrm{~A}$ total of 113 patients with a pre-operative myopia between -1.50 and -6.50 dioptres and an ablation diameter of $3.5-5.0 \mathrm{~mm}, 62 \%$ of whom had had both eyes treated, answered a questionnaire at the 36-month examination.

Halo problems were experienced always by $34 \%$ of patients in the group, $26 \%$ sometimes, and $40 \%$ never. Permanent night vision problems were experienced by $40 \% ; 30 \%$ reported them sometimes and $30 \%$ reported no problems at all. Sixty per cent never needed to use glasses after treatment, $30 \%$ sometimes did, and $10 \%$ always wore glasses.

The authors conclude on an optimistic note that $70 \%$ of patients thought the final results were very good; the remaining $30 \%$ experienced varying degrees of halo and night vision problems. ${ }^{18}$

\section{LASER IN-SITU KERATOMILEUSIS (LASIK)}

LASIK is a technique combining the advantages of lamellar corneal surgery (ie, under a corneal flap, therefore not disturbing Bowman's layer centrally) with the precision of excimer laser surgery. It can be used to correct moderate to high myopia between -2.00 to -20.00 dioptres, with fewer refractive complications.

The technique of performing excimer surgery under a raised flap and the term LASIK was coined by Pallikaris and co-workers. ${ }^{219}$ Current surgical algorithms may need modification to improve predictability. ${ }^{20-22}$ Stability of refraction after surgery requires further study but results are promising. LASIK must only be performed by experienced ophthalmic surgeons because of the possible complication of penetration of the globe and serious intra-ocular injury. Occasionally, the corneal flap may also become permanently displaced or lost. ${ }^{219-22}$

\section{PHOTOTHERAPEUTIC KERATECTOMY (PTK)}

PTK is an effective method of treating a variety of corneal disorders involving the corneal epithelium or anterior corneal stroma (box 7). Laser ablation of corneal lesions occupying the anterior stroma is a relatively safe means of treating several types of corneal scars and may occasionally offer an alternative to corneal transplantation. ${ }^{1823-30}$

Tuunanen and Tervo performed PTK in 39 eyes of 38 patients with various corneal diseases. ${ }^{28}$ Patients were grouped into four diagnostic categories: group 1 consisted of 11 eyes with postinfectious or post-traumatic corneal scars; group 2 consisted of 15 eyes with various corneal dystrophies or keratopathies; group 3 consisted of two eyes with recurrent corneal erosion; and group 4 consisted of 11 eyes with either primary or recurrent pterygia. Postoperative follow-up ranged from 3 to 15 months (mean 9 months). The goals of PTK (visual improvement, ocular comfort, or visibility for cataract extraction) were set individually for each patient and were achieved in 19 of 38 eyes (50\%); one patient was lost to follow-up

Best corrected visual acuity improved two or more Snellen lines in nine of 31 eyes $(29 \%)$. Both cases of recurrent corneal erosions were successfully treated. One eye lost two Snellen lines because of increased irregular astigmatism after PTK. In two eyes, corneal decompensation was observed 1 month after the operation, no other serious complications were observed. The excimer laser was combined with surgical abrasion or topical EDTA chelation, also with good results.

The authors concluded that the excimer laser is a valuable tool for treating anterior corneal disease. It can also be combined with other surgical methods such as surgical abrasion and chelation. Results could have been further improved with better patient selection criteria and by evaluating PTK on the basis of visual expectations and type and location of corneal pathology. ${ }^{28}$ 


\section{Conclusions}

Excimer laser surgery is an advancing field of ophthalmology whose use will greatly increase in the future. ${ }^{1831}$ Many patients are already happy with the results of PRK. ${ }^{18}$ The procedure is well tolerated by patients and is performed as an outpatient procedure. ${ }^{18}$ Current problems with photorefractive procedures include the absence of long-term data, the possibility of blurred vision due to corneal 'haze' (opacification), impaired night vision and haloes, and the lack of predictable results with high myopia. ${ }^{1} 8193132$

The authors would like to acknowledge the help and assistance of Mr Peter McDonnell, MRCP FRCS FRCOphth, and Professor Phillip Murray, PhD FRCS FRCOphth, of the University of Birmingham.

1 Seiler T, Mc Donnell PJ. Excimer laser photorefactive keratectomy. Surv Ophthalmol 1995;40:89-118.

2 Pallikaris IG, Siganos DS. Excimer laser in situ keratomileusis and photorefractive keratectomy Surg 1994;10:498-510.

3 Abrams D. Duke-Elder's Practice of refraction. London: Churchill Livingstone, 1978; pp 18892 .

4 Elkington AR, Frank HJ. Clinical optics. London: Blackwell, 1988; pp 116-21.

5 Epstein D, Fagerholm P, Hamberg-Nystrom H Epstein D, Fagerholm P, Hamberg-Nystrom $\mathrm{H}$ Tengroth B. Twenty-four-month follow-up of excimer laser photorefractive keratectomy for myopia. Refractive and visual acuity results. Ophthalmology 1994;101:1558-63.

6 Talley AR, Hardten DR, Sher NA, et al. Results one year after using the 193-nm excimer lase for photorefractive keratectomy in mild to moderate myopia. Am f Ophthalmol 1994;118:304 11.

7 Hersh PS, Patel R. Correction of myopia and astigmatism using an ablatable mask. $\mathcal{f}$ Refract Corneal Surg 1994;10:250-4.

8 Claoue C, ed. Laser and conventional refractive surgery. London: BMJ Publishing Group, 1996; surgery. London: BM-1 Publishing Group, 19

9 Sutton G, Kalski RS, Lawless MA, Rogers C. Excimer retreatrnent for scarring and regression Excimer retreatrnent for scarring and regression after photorefractive keratectom

10 Cherry PM, The treatment of pain following excimer laser photorefractive keratectomy. Oph excimer laser photorefractive kerat

thal Surg Lasers 1996;27:S477-80.
1 Gartry DS, Kerr Muir MG, Lohmann CP

1 Gartry DS, Kerr Muir MG, Lohmann CP, on refractive outcome and corneal haze afte photorefractive keratectomy. Arch Ophthalmo 1992;110:944-52.

12 Gartry DS, Kerr Muir MG, Marshall J. The effect of topical steroids on refraction and corneal haze following excimer laser treatment of myopia: an update. A prospective randomised double-masked study. Eye 1993;7:584-90.

$13 \mathrm{Kim}$ JH, Sah WJ, Kim MS, Lee YC, Park CK. Three-year results of photorefractive keratectomy for myopia. F Refract Surg 1995;11:24852.

14 Gartry DS, Kerr Muir MG, Marshall J. Excimer laser photorefractive keratectomy 18-month follow-up. Ophthalmology 1992;99:1209-19.

15 O'Brart DP, Lohmann CP, Fitzke FW, Smith SE, Kerr-Muir MG, Marshall J. Night vision after excimer laser photorefractive keratectomy: haze and halos. Eur $\mathcal{F}$ Ophthalmol 1994;4:43-51.

16 O'Brart DP, Corbett MC, Lohmann CP, Kerr Muir MG, Marshall J. The effects of ablation diameter on the outcome of excimer laser photorefractive keratectomy. A prospective, randomized, double-blind study. Arch Ophthalmol 1995;113:438-43.

17 Cherry PM. Removal of epithelium and scraping the underlying stroma as treatment for photorefractive keratectomy overcorrection or undercorrection of myopia. Ophthal Surg Laser 1996;27:S487-92

18 Hamberg-Nystrom H, Tengroth B, Fagerholm P, Epstein D. Patient satisfaction following photorefractive keratectomy for myopia. $\mathcal{F}$ Refract Surg 1995;11:S335-6.

19 Pallikaris I, Papatzanaki ME, Siganos D. A corneal flap technique of laser in situ keratomileusis. Arch Ophthalmol 1991;145:1699-702.

20 Salah T, Waring GO, el Maghraby A, Moadel K, Grimm SB. Excimer laser in-situ keratomileusis (LASIK) under a corneal flap for myopia of 2 to (LASIK) under a corneal flap for myopia of 2 to 83.

21 Helmy SA, Salah A, Badawy TT, Sidky AN. Photorefractive keratectomy and laser in situ keratomileusis for myopia between 6.00 and 10.00 diopters. $\mathcal{F}$ Refract Surg 1996;12:417-21.
22 Guell JL, Muller A. Laser in situ keratomileusis (LASIK) for myopia from -7 to -18 diopters. $\mathcal{F}$ Refract Surg 1996;12:222-8.

23 Cameron JA, Antonios SR, Badr IA. Excime laser phototherapeutic keratectomy for shield ulcers and corneal plaques in vernal keratoconulcers and corneal plaques in vernal ke

24 O'Brart DP, Muir MG, Marshall J. Phototherapeutic keratectomy for recurrent corneal abrasions. Eye 1994;8:378-83.

25 Moodaley L, Liu C, Woodward EG, O'Brart D Muir MK, Buckley R. Excimer laser superficia keratectomy for proud nebulae in keratoconus. Br 7 Ophthalmol 1994;78:454-7.

26 Ward MA, Artunduaga G, Thompson KP, Wilson LA, Stulting W. Phototherapeutic keratectomy for the treatment of nodular subepithelial tomy for the treatment of nodular subepithelial corneal scars in patients with keratoconus who are con $130-2$.

27 Thomann U, Meier-Gibbons F, Schipper I. Phototherapeutic keratectomy for bullous keratopathy. Br $₹$ Ophthalmol 1995;79:335-8.

28 Tuunanen TH, Tervo TM. Excimer lase phototherapeutic keratectomy for corneal phototherapeutic keratectomy for corneal 67-72.

29 Orndahl M, Fagerholm P, Fitzsimmons T, Tengroth $B$. Treatment of corneal dystrophies with excimer laser. Acta Ophthalmol 1994;72:235-40.

30 Migden M, Elkins BS, Clinch TE. Phototherapeutic keratectomy for corneal scars. Ophthal Surg Lasers 1996;27:S503-7.

31 Carson CA, Taylor HR. Excimer laser treatmen for high and extreme myopia. Arch Ophthalmo 1995;113:431-6.

32 Taylor HR, Guest CS, Kelly P, Alpins NA. Comparison of excimer laser treatment of astigmatism and myopia. Arch Ophthalmol 1993;111. 1621-6. 